DEPARTAMENTO DE PATOLOGIA E CLANICAS CIRGRGICA F OBSTFTRICA

Diretor: P'rof. Dr. Ernesto Antonio Matcra

\title{
CONTRIBUIÇĀO PARA O ESTUDO DA RAQUIANESTESIA PERIDURAL NO CÃO (")
}

\author{
CONTRIBLTION TU THE STLDY OF THE FPIDLRAL, ANAESTHESIA
}

IN THE DOG

\author{
F.. A. MATERI
}
A. V. Stopici.is
M. Anivlinziata
J. S. M. VEIGA
issistente
Assistentc
Assistente voluntário

A introducão de soluções anestésicas no canal vertebral, constitui métodos de anestesias espinhais.

() aulores velerinírios (Wright, Cinotr e (GikCiA), reconhecem duas variedades dislinlas desla aneslesia:

1 - sub-aracnoidea (raquianestesia sub-aracnoidea), na qual o anestésico é introduzido no espaço homônimo, difundindo-se no liquido céfalo-raquideano;

i - extradural, peridural ou epidural (raquianestesia extra, peri ou epidu. ral!, obtida mediante a injeção de substância ancstésica no espaço peridural, bloqueando as raizes nervosas raquideanas extradurais.

A literatura aponta Corning, em 1885, como o precursor da introdução "de soluções de cocaína no espaço extradural do canal vertebral de cães, com fina. lidades experimentais, e depois no homem, com a intençĩo de terapêutica neu. rológrica. Todavia, deve-sc, scgundo DocLIotTı, a Cathelin (1900) e a Lïwen (1010), a descriçäo do método de anestesia espinhal extradural, com objetivos cirúrựicos.

(aille e Chelle (citado por WrighT), foram os primeirou, ao que consta, a estudaren a aplicaçāo prática da aracstesia peridural no căa. Posteriormente, de acôrdo com ВRook, contribuem para a literalura, com observaçoues sûbre o en! rêgo déstc tipo de anestesia, nas virias espécites animais, os autores: - Retzgen, Paje: e P'izch (1925), Benesch (1926), McLeod e Fraik (1927), Götze,

(") Apresentado ao Il congresso l'an-Americano de Medicina Veterinária, realizado em Sïo l'aulo, de 3 a 10 de abril de 1954. 
Richter e Lagerlöf (1928). Brook, Golfincl, Corner 119:361. " nowamente Cuille e Chelle (1931).

A revisăo bibliografica à mão, sibure a aplicagĭo da raquianeslesia peridu. ral na especie canina, revela a existência de numerosos trabahos, dectacando-se

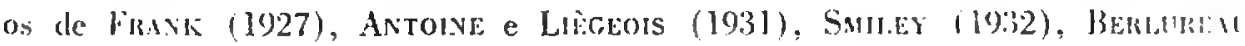
(1933), Brook (1935), Horkins (1936), Fliners, Martix e Byrit (1938), BeNesch (1939), Grafe e Schilzf 19.19), (iblas (1951), Moralfes (1952) e Midopils (1953).

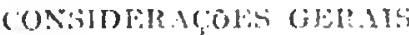

Espaço peridural -- O espaço quıe existe entre o saco dural c o caual isseto vertebral é denominado espaço cpidural ou peridural. Foi sierzi o primeiro a denominálo cspaso perílural, porquanto se trata de expaço circular que ahrange com toda a circunferência o saco dural. Outros aulores tambem adotam iste nome.

O interior do espoço i ocupado por lecido conectivo, rico em gordma, e rede venosa (plexo venoso peridural).

O tecido existente no espago peritural continnase com os de localizacino

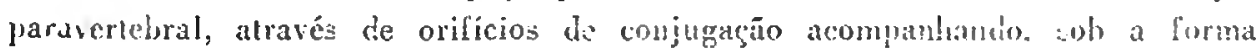

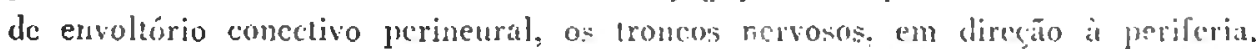

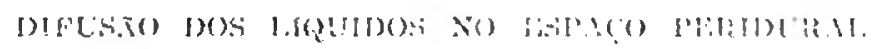

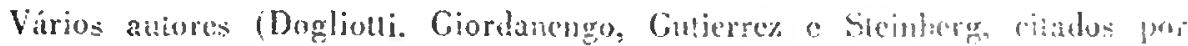

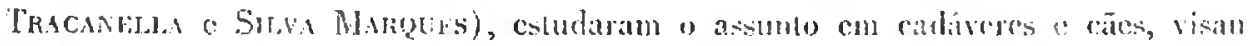

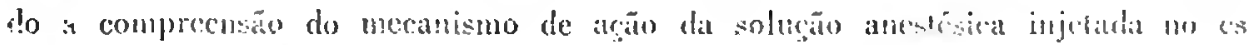
pa.o peridural. Concluiram que os líguidos injelados se difundem ao longo do

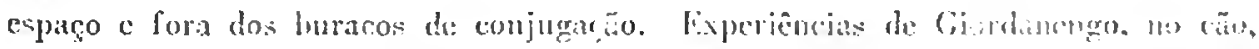

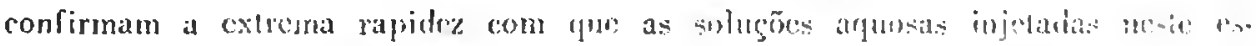

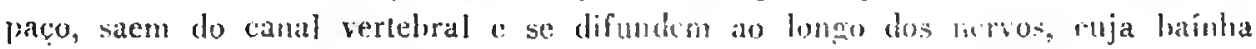

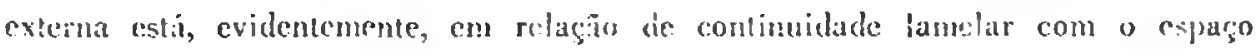
perichural.

Obecrvacoues con a finalidade de estudar a permeahilidude da duramater. mostraram que somento pequenas quantidades de anestésico altatusam o exojo

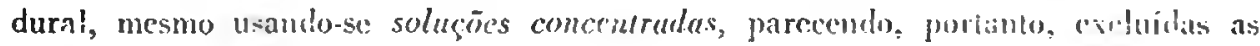

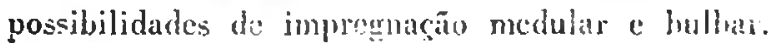

Dogtorto considera a anestesia feridural uma "anentesiat roncular em grande território", pela lacilidade com que us lijuides injeladus st: difundem. 


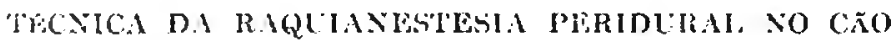

lirs/mmpinfal: -- I samos aguiha de $50 \times 10$, com o bisel menos aguçado, mis lenen corlanic. bute tipo de ponta reduz o periggo da perfuraçăo da dura. 1:1.4iry'.

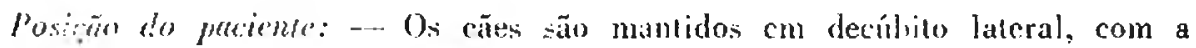
coluna lam flexionadia: flara éte fïn, os membros postariores seräo puxados pira a lremle. manlidos entre os anteriores que penuanceräu keguros cm sentick riposto.

The fir tersso: -..- l'amos sempre a via mediana. introrluzindo-se a agulha

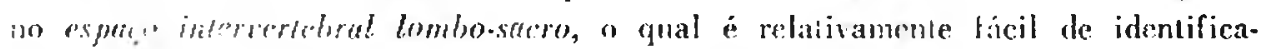
rár. prois Incaliza-se logo alrits do processo espinhoso da úlsina vértchra lom-

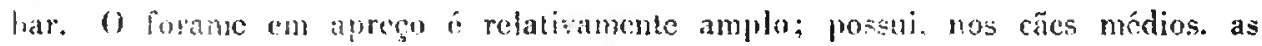

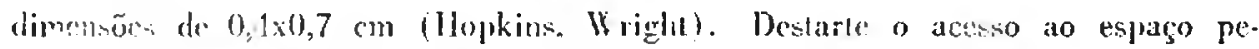

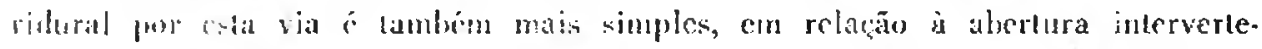

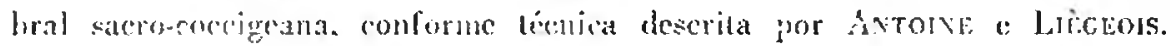

Guamin a ponta da agullea alcanga os ligamenos, sente-se resistencia, a qual

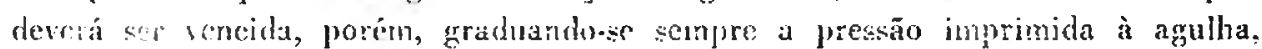
ate alingir o espago peridural.

Ante- de injetar a soluçäo ancestricica convem proceder a aspiragão com se-

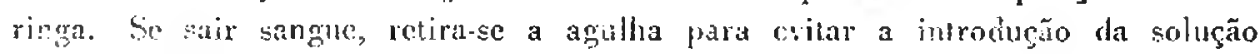
con uma das veias do plexo peridural. Caso sáia líquicio cófalo-raquideato. é comeniente retirar a dgutha para näo praticar una raquicuestesia sub-aracmoirkat.

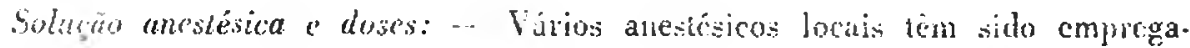
dos nas aliesicsias copinhais, a comecar pela cocaion; Cublot, em sua obra da-

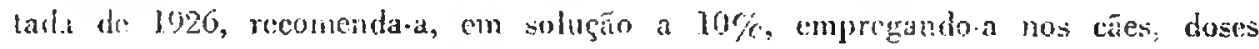
(10. $3 \mathrm{~mm}$ a 3 a cle

Cimon indica para a raquianestesia epidural dr cãcs, a talocaina a $5 \%$ ou a torcaina a $0.5 \%$, nas quanticiades variácis de 3 a $20 \mathrm{ml}$, conforme o talhe.

SuHer usa também a tutocaina a $1 \%$, con resultados incertos, e a $2 \%$, main eficientamente.

Wirsint olutém resultados satisfatórios com a tutocaina a $2 \%$, ascociada à adirenaliuga a $0,00.5 \%$

A subutancia, codavia, mais larsymente usada, é a Norocaina ineocaina, proraina, alocaina, etoiaina, sincaina, escurocaina), devido a baixa toxicidade e en alto poder ancstésico. 


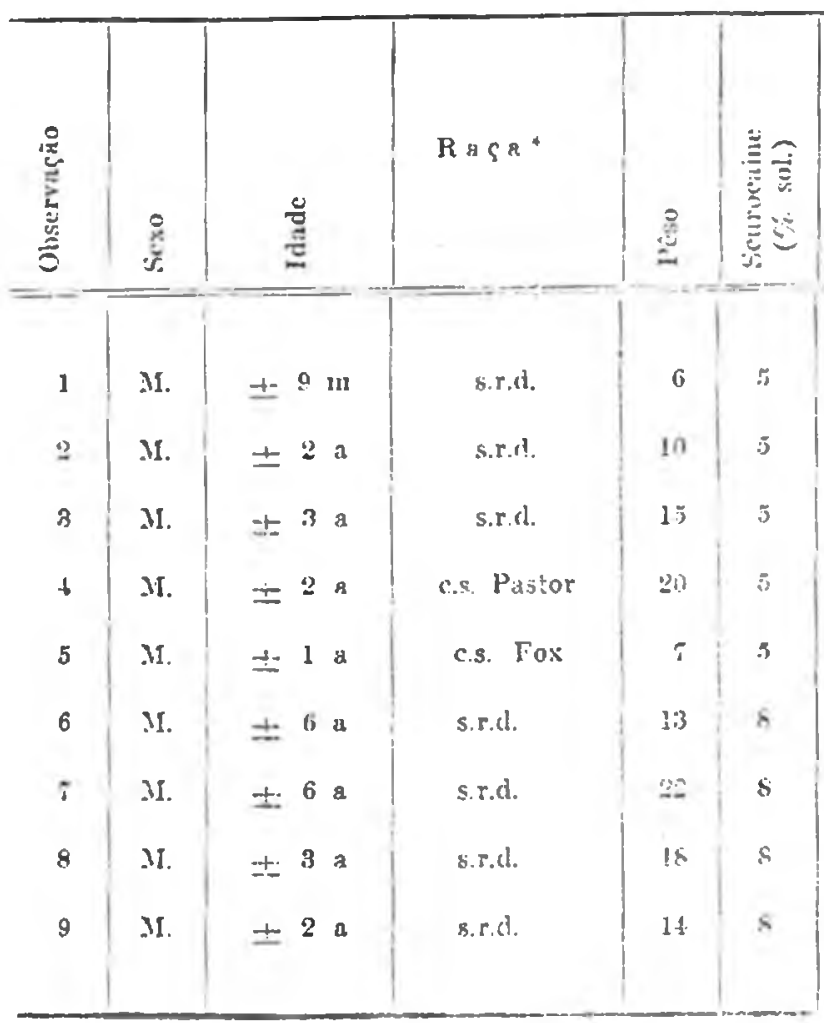

- s.r.s. $=$ sem roca ciefinicos

s.s. = com sungue 


\section{QUADRO I}

\begin{tabular}{|c|c|c|c|c|c|c|}
\hline $\overrightarrow{\mathrm{E}}$ & 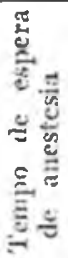 & 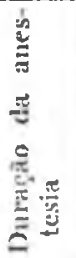 & 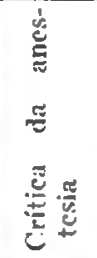 & 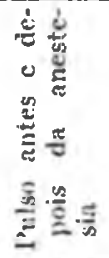 & 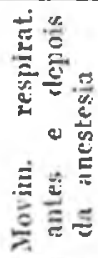 & $O c u r r b c i a s$ \\
\hline I & 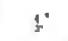 & $90^{\circ}$ & itima & $1 \cdot 21)-1 \div 0$ & 年 & \\
\hline 2 & 5 & $90^{\circ}$ & itima & $! U-9 !$ & & \\
\hline 3 & $\pi$ & $90^{\circ}$ & ólina & N:)-80 & $20-20$ & \\
\hline+ & $5^{\circ}$ & $100^{\circ}$ & itima & $11:-110$ & $36 \mathrm{i} 36 \mathrm{j}$ & \\
\hline 1 & $r$ & $60^{\prime}$ & regular & $1(0 i) \cdot 190$ & $20-20$ & - Inestesia incompleta. \\
\hline 2 & 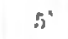 & $60^{3}$ & regular & $30-80$ & .93 .29 & Anestesin incompleti. \\
\hline 5 & 3 & $90^{\circ}$ & étiv:a & $5 ! 1)-80$ & $2=-20$ & \\
\hline 4 & 2 & $80^{\prime}$ & ótims & $120-1 \geq 0$ & 30.30 & \\
\hline 3 & 2 & $80^{\circ}$ & oting!a & $110-110$ & $20)-20$ & \\
\hline
\end{tabular}


QLADRO II

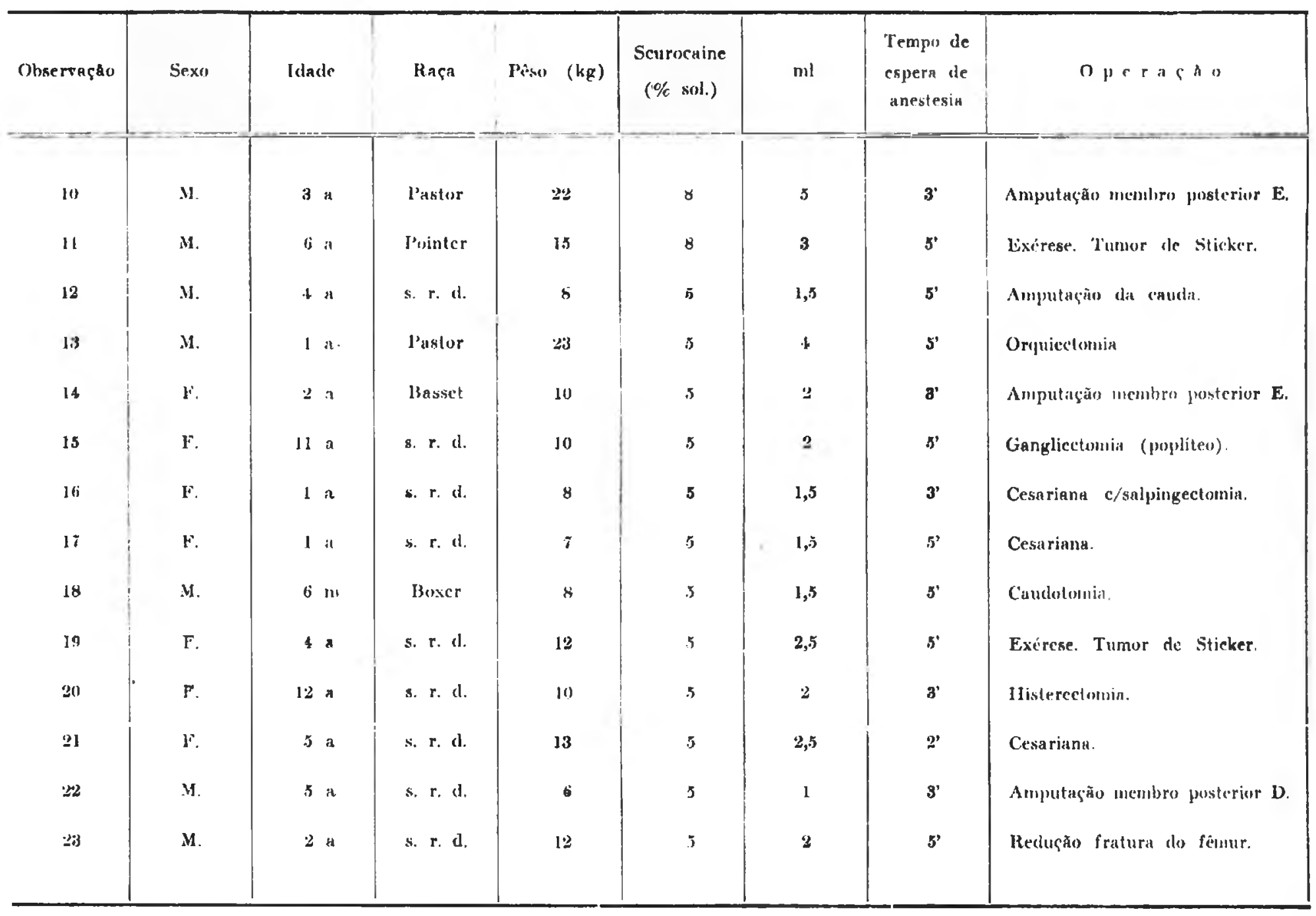



Pretendemos continuar os entudos com pesquisas sôbre a funçăo renal e sôbre o fígado, a fim de confirmar a ausência dr. alterações nestes órgãos.

Igualmente, exames anátomo-patológicos de cães anestesiados experimentalnente, não revelaram lesóes medulares ou em outras partes do canal vertehral.

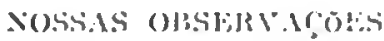

Nossas observasörs experimentuis, referem-se aos casos que aprescntamos no quadro I, havendo scmpre anestesia e volta integral da sensibilidade. Na referencia $1 " 5$, verificamos que a anestesia foi incompleta; o animal manteve certa mobilidade, demonstrando sensibilidade decorridos sessenta minutos após a apli(açio. A dose neste: caso, parecen-no: insuficionte.

() me:mo ocorren no caso n" 6 .

Ali: a presente data, efcuamos ritorar? reperescoes, adotando a anestesia em apresco. conforme sumário no quadro II. O animal cilado no caso $\mathrm{n}^{\circ} 16$. faleceu logo após o ato cirúrgico, prosàvelmente em consequência das condiçôes gerais peissimas, em quic se apresentava.

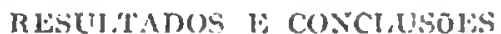

Pellas nossas observeçócs sôhre a raģuianestesia peridural, no espaço lombo. cạrro, cul cáes, ('mpregando solução de Novocaína a jớc, com adrenalina, ou jura a $8 \%$ (Scurocaine - Rhodia), verificamos:

1 - P'cruenas doses. antes nunca núlizadas (1. ml para cada 5.6 quilos de pêso vivo), foram suficientes para detcrminar anestesid perleita e completa $\mathrm{cm}$ toda a profundidade da caudi. dos membros posicriores, da re. giāo prineal, da região dorsolombar, dos órgãos genilais e das paredes - da cavidade ahdoninal, siluadas caudalmente à reçis̆o umbilical.

2 - Relaxamento de tôda a musculatura da regiä́o ancitesiada.

3 - Abreviasão no tempo de espera da anestesia (3) a 5 minutos).

4- Anestesia mais duradoura, em relaģ̃a aos dados publicados em oulros Irahalhos (unia c meia hora a duas horas);

5 - Pulsaçóes e movimentos respiratórios sem alteraroũes durante, on após a fase ancstésica.

6 - Roas condições durante o ato operatúrio, nas intervençóes praticadas. Ansência de acidentes durante a anestesia. Pós-operalório, sem complicacoües devidas à anestesia. 


\section{SUMMARY}

Studies on the application of small doses of procainc (Scuroraine 5 and 3 per cent) in the peridural (epidural) ancsthesia in dogs, have heen carried out.

This technique has shown very good results as far as anesthesia conditions are concerned and a lot of advantages when in comparison to the findings of others authors who have worked on the same subject.

\section{BIBLIOGIRAFIA}

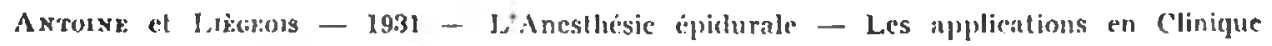
Caniue. Ann. Med. T'it. 337-45

Baoenna, G. c Colombo, C. - 1952 - Lianestesia epidurale ciudide sacrocoerigrax ed intercoccigea. Método delecione per le interuenti sullapparato genitale nel gatto. Zootecmate e I'eterinaria, Milano, 7(1-5):1.4-50

Braser - 1999 - 1.anesthesic en obstétripue et gynicologic vélérinaires. "Cit." Ann. Med. Fet. $82-6$

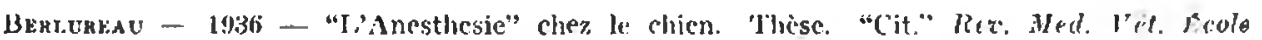
1'. Ilfort, 112:7.49

Вноок, (i. 13. - 1935 - Spinal (epidural) anesthesia in the Domestic Auinals. Pet. Rec. $15(19): 549-53 ; \quad(23): 659-67$

BrHxy, J. M. - 1938 - Ancsthesia in Veterinary J'ractice - I'et. Rec. 50(48):1161-8

Canot, I', I. - 1926 - Précis de Chirurgie Vétérinuire: 61, sème. dol. - Paris, Vignot frìres

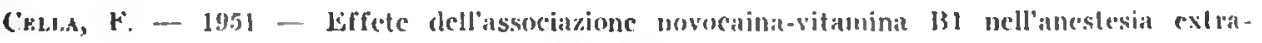
lurale. Alli. Noc. Jlul. sciknze zut. $5: 11: 2-1 ;$

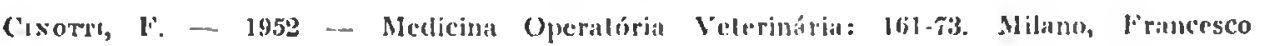
Vallardi

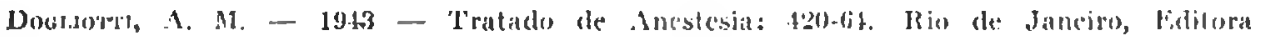
steitntifica

Frunems, G. - 1939 - Anesthesia in small inimals. Justi. Hef. Juur. 14:1-42-6

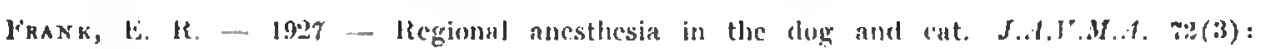
; $3: 36-10$

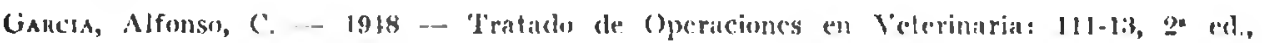
Madrid, Jamprental liosscit

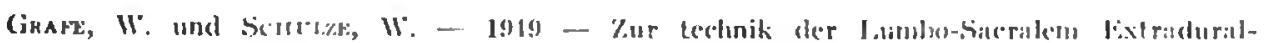
anaesthesic beimbund. Berl. und Minch. Tiesërs. Woch., 10:[1(1)-1

Jopkiss, G. S. - 1980 - The Correlalion of Analonry and bipdural Anestluesia in

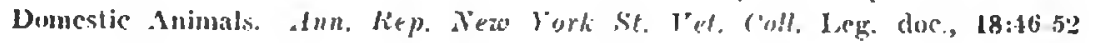


Lacnolx, J. V. Rsske, Wr. II. - 19.49 - Canine Surgery: 105-1t; North Amer. V'et.

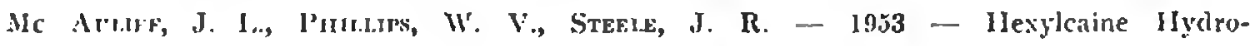
diforide: in Velcrinary Surgery. I'et. Hed., 7:261-7

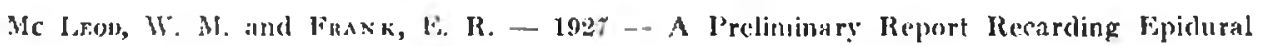

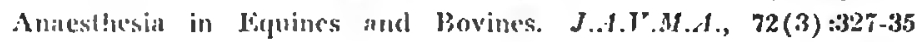

Matrina, 1\%. A. - 19152 - I'roslatertomin peln V'iat Jerincal no Cão. 'rèse. Săo l'aulo

Mink, 11. J. - 1919 - I’raclical Veterinary I'larmacology - Matéria Médica and 'lherapeuties: 21\%, Gith cdi., 1.onchon, Bailibere, 'lindibll and Cox

Morai.es, I. 1'. - 1952 - Anestesiat subchural en el perro. Ret. Fur. Med. Ves. $y$ Zaot., Bogotí, 105:66-88

Niwros, 0. M. - 1951 - Nuevas Adquisiciones sobre aplicación de la anestesia regional cpidural candal. Girer. Jet., Buenos dires, 18(7t):25.1-61

O'coxxon, J. J. - 1950 - Dollar's veterinary surgery: 2I3-t, ath col. I.ondan, Baillicre, 'liusdall and cox

IRrmbil,., W. K. - 1953 - Caudal Anesthesia in Canine Surgery. Jer. Sul. Jour, 18

Simuks, 11. 1). - 1932 - Some Further Experiments wilh I.umbar Anesthesia in Cinines. I.A.I.M...1., 33:560-78

Tuacispira, O. c Sird Marques, J. - 1939 - Anestesia peridural. Arq. Cir. Clin. Bxp. Supl. 3: $139-266$

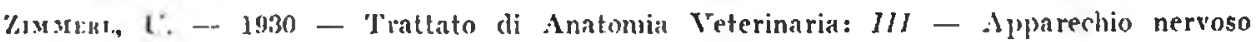
(meningi) : 419-60, Milano - Francesco Vallardi

WrarrT, 1. G. - 10.52 - Veterinary Anacsthesia: 51-7, 81-5, 3th ed. London, Baillière, 'iundall and Cox 